\title{
A descriptive model of the cigarette epidemic in developed countries
}

\author{
Alan D Lopez, Neil E Collishaw, Tapani Piha
}

\begin{abstract}
It has been estimated that cigarettes are the cause of the deaths of one in two of their persistent users, and that approximately half a billion people currently alive $-8 \%$ of the world's population could eventually be killed by tobacco if current smoking patterns persist. Despite this pandemic, tobacco consumption continues and is increasing in many countries, especially in Asia and in Southern and Eastern Europe. A major factor affecting public awareness of the substantial health hazards of tobacco use is the three- to four-decade lag between the peak in smoking prevalence and the subsequent peak in smoking-related mortality. Based on nearly 100 years of observations in countries with the longest history of widespread cigarette use, a four-stage model of cigarette consumption and subsequent mortality among men and women is proposed. From the model it is clear that, during certain periods of evolution of this epidemic, it is to be expected that smoking-attributable mortality will rise at the same time that smoking prevalence might be decreasing. This is because current mortality is most closely related to previous, not current, levels of cigarette consumption. Broad geographic classifications of regions are given, according to the stage of the epidemic that they are currently experiencing. Tobacco control policy implications for countries at each of the four stages of the cigarette epidemic are also discussed.
\end{abstract}

(Tobacco Control 1994; 3 : 242-247)

Tobacco or Health

Programme,

World Health

Organisation,

Geneva, Switzerland

AD Lopez

NE Collishaw

Action Plan for a

Tobacco-Free Europe,

WHO Regional Office

for Europe,

Copenhagen,

Denmark

T Piha

\section{Introduction}

Cigarette smoking has been identified as the single most important cause of premature death in developed countries, and is already of major public health concern in many regions of the developing world. Recent estimates suggest that about two million people die each year in the developed world from smoking, half of them before age $70 .{ }^{1}$ The risk of death from smoking - about one in two among persistent smokers $^{2}$ - far exceeds that of any other addiction, exposure or injury, something which is generally not appreciated by either smokers or non-smokers. In part, this lack of understanding about the extraordinary risks associated with smoking reflects public confusion about how these risks evolve and, in particular, about the long delay between the onset of persistent smoking and the massive increase in death rates which it produces some decades later. Similarly, smokers are often not aware of the rapid decline in risk which occurs soon after cessation, most notably from coronary heart disease. ${ }^{3}$

Information on cigarette consumption and the patterns and trends of the major diseases associated with it is available for many countries, covering a period of almost 100 years. While the reliability and availability of data vary from country to country, there is now sufficient evidence to categorise the various phases of the tobacco epidemic in a way which might be more informative for public health policy. One such categorization is proposed in this article.

This is not the first attempt to model the cigarette epidemic. Kunze, for example, has proposed a two-phase model of the relative evolution of smoking prevalence among physicians compared with the general population. ${ }^{4,5}$ According to this formulation (which would seem to be well supported by historical data from developed countries), ${ }^{6}$ smoking rates among doctors initially exceed those of the general public due largely to their greater disposable income and their ignorance of, or indifference to, knowledge about the health hazards of smoking. Subsequently, as the dangers of smoking become increasingly apparent, physicians give up smoking earlier and in greater numbers than the general population with the result that their prevalence falls below that of the population at large.

Clearly, there are many distinct characteristics of the epidemic which could be used to define phases for policy purposes, and hence what is presented here is merely one representation of the epidemic transition which all countries have, to some degree at least, unfortunately begun. It is unlikely, however, that the principal defining characteristics of each phase would alter, irrespective of the number and timing of transitional states which one might choose to identify. 
A model of the cigarette epidemic

Transition through the various stages of the tobacco epidemic can be effectively characterized by changes in three variables:

- prevalence (the percentage of the adult population who smoke regularly);

- consumption (the amount smoked per adult in a given period, eg, per year);

- mortality due to smoking (numbers of deaths attributed to smoking, by age, sex, and classified cause of death).

\section{MEASURING TOBACCO USE}

Prevalence and consumption are clearly interrelated. Both are useful for assessing the extent to which smoking has become entrenched in a population, although prevalence is probably a more relevant marker for public health purposes. The proportion of adults who smoke regularly is a simple, timely, and powerful indicator of the diffusion of the smoking epidemic. Specific prevalence levels among defined population sub-groups (eg, sex, age, social class, profession) indicate the progressive and differential adoption of smoking behaviour and are probably the most relevant statistics for tobacco-control programmes. Consumption, on the other hand, varies with prices and incomes. Smokers typically increase consumption with increasing disposable income to attain a daily average somewhere between 15 and 30 cigarettes. There is, however, a wide range of daily consumption and certainly it is reasonable to assume that, at least among smokers whose consumption is low (ie, less than 8-10 cigarettes a day), affordability, in addition to other factors, is of importance. In short, while the pattern and level of cigarette consumption are clearly relevant in categorising the epidemic, prevalence is likely to be a much better indicator of population exposure to the hazards of tobacco.

Prevalence data, however, have their limitations. To begin with, these data can only be gathered through censuses or population surveys, and long series of such data, useful for monitoring the progress of the epidemic, are not available in comparable form for the majority of countries. Few countries have reliable smoking prevalence estimates prior to about 1960, thus restricting historical analysis of smoking patterns (this can be overcome to some extent from birth-cohort analyses which reconstruct the smoking histories of cohorts and thus provide valuable information about smoking patterns in previous years). ${ }^{7}$ Survey data have also been found to underestimate both the number of smokers as well as the number of cigarettes smoked, particularly in younger age groups. ${ }^{8}$

Consumption figures derived from tobacco sales data, and typically reported as the weight of tobacco or the number of cigarettes consumed per adult 15 years and older, have the advantage of being available in comparable form for a large number of countries over long periods of time. They are also likely to provide a more accurate measure of total tobacco consumption. On the other hand, overall consumption levels derived in this fashion do not provide highly policy-relevant information about consumption by different categories or classes of the population. Prevalence data from surveys are indispensable for this. Both consumption and prevalence data are necessary for a full understanding of population exposure to the hazards of tobacco, and both are used here to define phases of the epidemic.

\section{MONITORING THE HEALTH EFFECTS}

Smoking causes mortality through a variety of diseases, principally several sites of cancer, major vascular diseases, and chronic lung diseases. The delay before death rates among smokers begin to rise varies from one disease to another. For lung cancer, the health effects are generally not apparent until 20 or 30 years after smoking becomes widespread in a population. Indeed, death rates from lung cancer generally do not reach their maximum until 30 to 40 years after the peak in smoking prevalence.

In the model shown here, lung cancer death rates have been used as an index of total smoking-attributable mortality. The use of the lung cancer dose-response relationship to estimate smoking-attributable mortality from other diseases is obviously open to question. For chronic obstructive pulmonary disease and certain sites of cancer, where the pathological mechanism of organ damage due to cumulative exposure to cigarette smoke is similar to that of lung cancer, the estimated dose-response relationship suggested by the model may be quite plausible. Declines in lung function are already apparent in smokers by the age of 25-30 years, and pathologically definable pulmonary emphysema typically emerges among smokers in their 50s. ${ }^{9}$

On the other hand, there is evidence to suggest that the lethal effects of smoking on major vascular diseases may occur somewhat earlier in life than for lung diseases. The excess mortality of smokers from coronary heart disease compared with nonsmokers is substantially greater (two-fold excess) in middle age (40-69) than at older ages. Furthermore there is a rapid decline in excess risk of death from major vascular diseases with smoking cessation, and among those ex-smokers who consumed less than a pack of cigarettes per day, death rates from coronary heart disease are comparable to those of lifelong nonsmokers after about 10 years. ${ }^{10}$ Conversely, for lung cancer and chronic lung diseases, a comparable reduction in risk among exsmokers takes much longer. ${ }^{3}$

Given the important role of vascular diseases in total smoking-attributable mortality, at least in developed countries, the net effect of these varying lag-times may well mean that the model presented here, which uses lung cancer as a guide to the overall health consequences of smoking, might underestimate smoking-attributable deaths earlier in the epidemic and possibly overstate the number of deaths in populations where major vascular diseases have been declining for some time. 
Other scenarios are possible, however. Coronary and vascular disease are caused not only by smoking, but also by hypercholesterolemia, hypertension, and possibly other risk factors as well. ${ }^{10}$ There are different distributions of these risk factors across cultures, as well as differential interactions with smoking. Nonetheless, there is a broad similarity in the evolution of smoking prevalence and vascular disease mortality rates in countries that have the longest experience with widespread cigarette use.

\section{Characteristics of the model}

The relationship between smoking prevalence and smoking-attributable mortality is depicted in the figure, which summarises the various phases of the smoking epidemic. Four distinct stages are proposed in the model, although different countries will obviously exhibit different variations of this general picture. The principal characteristics of each stage are summarised below.

\section{STAGE I}

This essentially defines the very beginning of the smoking epidemic in a population. Male prevalence is comparatively low $(<15 \%)$; female prevalence, largely because of sociocultural factors which hitherto have discouraged smoking among women, would rarely exceed $10 \%$, and may well be less than $5 \%$ in many cases. Per capita (adult) consumption is also relatively low, generally less than 500 cigarettes per adult, most of which are smoked by males. Death and disease due to smoking are not yet evident.

This initial phase may be relatively brief, perhaps one or two decades, as smoking becomes socially acceptable and tobacco control strategies remain underdeveloped, with priority being given to reducing malnutrition and the burden of infectious diseases. By the end of it, however, male prevalence is rising rapidly with some male deaths attributable to tobacco, but very few. During this phase, lung cancer is extremely rare with levels in most cases comparable to those observed among contemporary non-smoking populations.

\section{STAGE II}

During this phase of the epidemic, which may span two to three decades, prevalence of smoking among men continues to rise rapidly reaching a peak somewhere in the range of $50-80 \%$. The proportion of ex-smokers is relatively low. Smoking prevalence among women typically lags behind that of males by one to two decades, but is increasing rapidly. Smoking prevalence may be similar among different socio-economic classes and, if anything, may even be slightly higher among the upper classes. Per adult consumption of cigarettes varies between about 1000 and 3000 per year, the majority of which are still consumed by males (among whom annual consumption would probably be in the range of $2000-4000$ cigarettes).
Tobacco control activities during this phase are generally not well developed. Education and information about the hazards of tobacco are unsystematic and sporadic. Lack of public and political support may prevent the successful implementation of tobacco control measures, in part because the risks of tobacco use may still not be widely understood. ${ }^{11} \mathrm{By}$ the end of this phase tobacco use is already causing about $10 \%$ of male deaths, but comparatively few in women. Male lung cancer rates would have risen 10 -fold, from about $5 / 100000$ to $50 / 100000$ (age-standardised onto the World Health Organisation (WHO) "European" population) while among females, the rise is likely to have been much more modest, perhaps reaching 8-10/100000.

\section{STAGE III}

Male prevalence begins to decline, quite possibly after exceeding $60 \%$ for an extended period, to around $40 \%$ by the end of this phase, which may last for three decades or so. Prevalence tends to be lower among middleaged and older men, many of whom have become ex-smokers. More importantly, the end of Stage III is characterised by an initial decline in female prevalence following a plateau which may be even longer than that proposed for men. Since knowledge about the health hazards of tobacco is generally widespread by this time, the peak in female prevalence is likely to be considerably lower than that for males. Experience from the US, UK, Canada, and other countries where smoking has been common among women for some time, suggests that the maximum prevalence for women is around 35-45\%. There is also likely to be a marked age gradient in female prevalence with something like $40-50 \%$ of all young women being regular smokers, but with relatively few $(<10 \%)$ smokers among women above $55-60$ years of age. By this point of the epidemiological transition, male prevalence may well be only about five percentage points higher than for females. Declines in prevalence would generally be significantly greater among the more highly educated as they respond more favourably to health promotion campaigns about the hazards of smoking.

On average, adult male consumption might be expected to vary between about 3000 and 4000 cigarettes per year, being towards the lower end of this range at the end of the period.* Consumption among women would be lower, probably in the range of $1000-2000$ cigarettes per woman per year.

* The increase in average consumption at a time of decreasing prevalence reflects the tendency for smokers, on average, to consume more cigarettes as this stage advances. In the UK, for example, adult male consumption of manufactured cigarettes varied between 3300 and 4000 per year between 1950 and 1980, smoking prevalence among men declined from $62 \%$ to $42 \%$, and consumption per smoker (male) per week increased from 100 to $150 . .^{12}$ In large part, this effect probably arises more due to lighter smokers quitting than to a significant increase in consumption among heavier smokers. 
Perhaps the most dominant characteristic of this period is the rapid rise in smokingattributable mortality, with the level for males rising from about $10 \%$ of all deaths to about 25-30\% within three decades. In middle age (35-69 years), the proportionate mortality due to tobacco is even higher (about one in three deaths). Female deaths due to tobacco are still comparatively low (around $5 \%$ of all deaths), but rising. By the end of this phase, male lung cancer rates (age-standardised) would have peaked at around 110-120/100000. Death rates from the disease among women would also have risen sharply, to levels around 25-30/100000.

Conditions for successfully enacting and implementing a comprehensive tobacco control law are more favourable. ${ }^{13}$ Smoke-free public places and transport are among the standard measures but smoke-free workplaces are not yet common. Systematic programmes on tobacco are often initiated in schools. The media play a key role in whether comprehensive tobacco control policy based on legislation can be introduced. By this time, smoking is changing from being socially acceptable behaviour to socially abnormal behaviour.

\section{STAGE IV}

Smoking prevalence for both sexes continues to decline more or less in parallel, but only slowly. Some $20-40$ years after reaching its peak, female prevalence might only have declined $10-15$ percentage points or so, and would typically be around $30 \%$. Male prevalence might be expected to be slightly higher, perhaps $33-35 \%$. Meanwhile, the rise in male mortality from smoking would be expected to peak early in this period, possibly at around $30-35 \%$ of all deaths, being as high as $40-45 \%$ of deaths in middle age. Within a decade or so after reaching this peak, proportionate mortality from smoking would fall below $30 \%$, and progressively decline thereafter. It would also not be unreasonable to expect lung cancer rates among men to have fallen by about $20 \%$ from their peak, within one or two decades into this phase.

Conversely, female deaths due to smoking would be rising rapidly as the full health effects of women's previous smoking patterns become evident. However, because their cumulative exposure would have been significantly less than males, their smoking-attributable mortality will be proportionately lower. About two to three decades into this phase, deaths due to smoking among women could be expected to peak at around $20-25 \%$ of all deaths, significantly lower than what men experienced and occurring about 20 years later. Thereafter, smoking-attributable mortality for both sexes would progressively decline.

Smoke-free personal environments become a key issue in this phase. People demand a smoke-free work-site, and legislation and work-site policies to that effect become feasible. Policies need to address the issue of nicotine-dependent smokers who would like to quit but are unable to do so. Social differences in smoking prevalence persist, and may even widen. Continued change in the social climate is crucial.

\section{Discussion}

The most important feature of this model is that it clearly shows the three- to four-decade lag between the rise in smoking prevalence, and the rise in smoking-attributable mortality. For countries in Stages I and II, it would be dangerous and erroneous to ignore tobacco as a public health problem because death rates due to smoking are low. If smoking prevalence is increasing, smoking-attributable mortality will inevitably increase three to four decades later, during Stages III and IV. The occurrence of simultaneously increasing mortality and decreasing prevalence among men in Stage III, and among women in Stage IV, has led to substantial confusion about, and underestimation of, the health hazards of smoking. The explanation is clear from the model depicted in the figure. Smoking-attributable mortality is most closely related to smoking patterns 30 or more years previously, and not to current prevalence.

As with all other epidemics with a major behavioural component, the exact timing, duration, and magnitude of the smoking epidemic will vary significantly from one country to another. The model proposed here is thus a very general categorisation of the transition and does not exactly describe the experience of any one country. For some countries, such as Australia, Austria, Belgium, Finland, Ireland, the Netherlands, New Zealand, Sweden, and the UK, the model is historical in nature. These few countries have experienced all four stages of the cigarette epidemic, and are now enjoying declines in smoking-attributable mortality, at least among men. Most other countries of Western Europe, along with Canada and the US are nearing the end of Stage III or passing into Stage IV. These countries share a common history of having passed through Stages I and II and, for some, even parts of Stage III, before the serious health consequences of smoking came to be known and widely understood in the 1950s and 1960s. Thus, only late-stage prevention has been possible. Through the success of tobacco control strategies, it has been possible to slow down the growth of tobacco-induced mortality from what it otherwise might have been. Still, tens of millions have already been killed by cigarettes in industrialised countries and, even under the most optimistic of tobacco-use reduction scenarios, tens of millions more will die from tobacco use before the epidemic runs its course.

Other countries, however, now have the advantage of knowing the serious health consequences of smoking and can undertake effective preventive interventions at earlier stages of the epidemic. They can prevent history from repeating itself by taking strong public health measures to arrest the growth in tobacco consumption during Stage I or Stage II of the epidemic. The earlier these inter- 


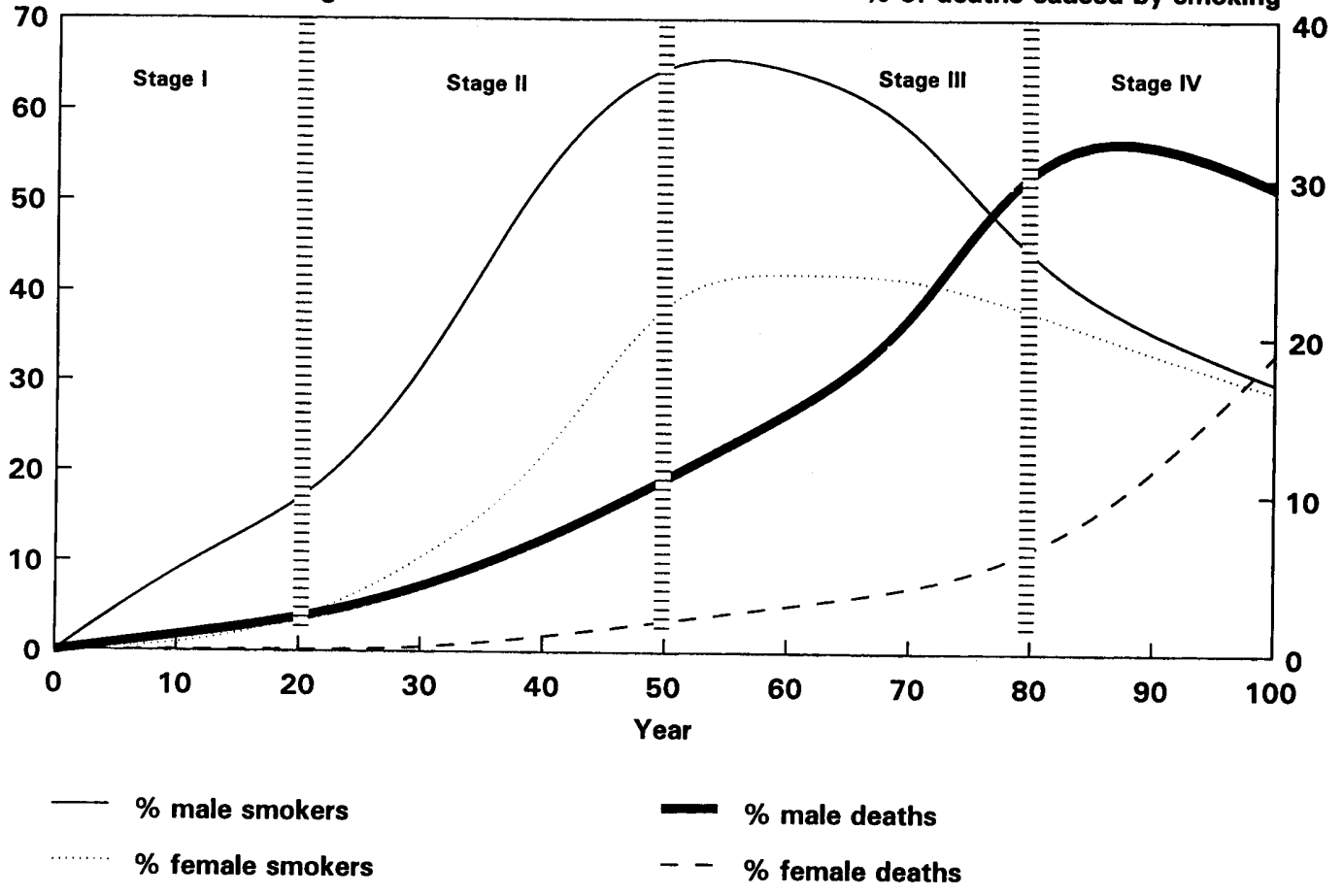

Figure $A$ model of the cigarette epidemic

ventions are undertaken, the more effective they will be, and the more premature deaths will be avoided.

Singapore is one country that has clearly demonstrated the advantages of early intervention. Singapore began implementing tobacco control policies as early as 1971 , with a partial ban on tobacco advertising. More and more elements have been added to the policy over the years, to the point where Singapore now has one of the world's strongest and most effective tobacco control policies. Besides the ban on advertising which has now become a total ban, other elements include effective protection from involuntary exposure to tobacco smoke, health warnings on packages, high tobacco taxes, a ban on incoming dutyfree sales of cigarettes, a prohibition on sales to minors, and strong programmes of health promotion and health education. In 1978, male smoking prevalence in Singapore was $42 \%$ and female smoking prevalence was $9.5 \%$, among the population 15 years of age and over. ${ }^{13}$ Smoking-attributable mortality was not apparent for either sex. In other words, in the 1970s Singapore was in the early part of Stage II of the cigarette epidemic. The effect of Singapore's tobacco control policies has been to limit male smoking prevalence to a peak of $42 \%$ in 1978 , lower than had been experienced elsewhere, and to subsequently reduce prevalence among men aged 18-64 years to $33 \%$ in 1992. Smoking prevalence among women aged $18-64$ years fell to $3 \%$ in $1992 .{ }^{14}$ Consumption of cigarettes fell from 2650 cigarettes per person aged 15 and over in 1980 to 1650 in 1990. Early Stage II intervention has thus prevented thousands of smoking-attributed deaths from ever occurring in Singapore.

Even greater success could be achieved by effective tobacco control measures being undertaken earlier in the epidemiological transition. Many developing countries, primarily in sub-Saharan Africa, are currently in Stage I and, by undertaking preventive action now, can still prevent the cigarette epidemic from becoming a major cause of death in the future. Other countries, be they in Stage II of the tobacco epidemic, such as China, Japan, and other countries of Asia, Latin America, and North Africa - or Stage III, characteristic of Eastern and Southern Europe - or even those in Stage IV, will need to initiate and/or sustain preventive measures if they are to halt the epidemic's progress and thus prevent tens of millions of unnecessary premature deaths from occurring some decades hence.

\section{Conclusions}

The arithmetic of the tobacco epidemic is simple and stark. Cigarettes kill half of their lifelong users. In countries where two-thirds of men and $40 \%$ of women smoked in the 1960 s, one-third of all male deaths and $20 \%$ of female deaths in the 1990 s are due to smoking.

Countries with lower rates of smoking prevalence need to act quickly to prevent smoking prevalence from rising, and to reduce it further. For, in the absence of a truly comprehensive national tobacco control programme, and in the absence of significant social pressures which discourage tobacco use, this epidemiological transition, with the tens of millions of premature deaths which result from it, is virtually certain to happen.

1 World Health Organisation. Smoking and premature adult mortality. World Health Statistics Annual, Geneva: WHO, 1990; 29-30. 
2 Peto R, Lopez AD, Boreham J, Heath C, Thun $M$. Mortality from tobacco in developed countries: indirect estimation from national vital statistics. Lancet 1992; 339. 1269-78.

3 US Department of Health and Human Services. The health benefits of smoking cessation A Report of the Surgeon General, 1990. US Department of Health and Human Services, Public Health Service, Centres for Disease Control, Centre for Chronic Disease Prevention and Health Promotion, Office on Smoking and Health, 1990. (DHHS Publication No (CDC) 90-8416.)

4 Kunze M. Current smoking habits in Europe. Presented at the European Conference on Tobacco Priorities \& Strategies, organized by the International Union Against Cancer and the Dutch Foundation on Smoking and Health, 1-3 November 1989, The Hague, The Netherlands.

Davis RM. When doctors smoke. Tobacco Control 1993; 2 : 187-8.

6 World Health Organization. World No-Tobacco Day, Press Kit (Fact Sheet). WHO, Geneva: WHO, 1993; 2 .

7 Harris J. Cigarette smoking among successive birth cohorts of men and women in the United States during 1900-80. f Natl Cancer Inst 1993; 71:473-9.
8 Collishaw NE. Cigarette consumption in Canada, 19811986. In: Aoki $M$ et al, eds. Smoking and health 1987. Amsterdam: Elsevier, 1988, pp 701-2.

9 US Department of Health and Human Services. The health consequences of smoking : chronic obstructive lung disease. $A$ report of the Surgeon General. US Department of Health and Human Services, Public Health Service, Office on Smoking and Health, Rockville, Maryland, 1984.

10 US Department of Health and Human Services. The health consequences of smoking: cardiovascular disease. $A$ report of consequences of the Sur Services, Public Department of Health and Smoking and Health, Rockville, Maryland, 1983. (DHHS Subling and Health, Rockville, Maryland, 1983. (DHH Publication No (PHS) 84-50204.)

11 Piha $T$. Tobacco control activities of the World Health Organization in Europe. In: Richmond R, ed. Interventions for smokers: an international perspective. Williams \& Wilkins, New York, 1994; 323-47.

12 Wald $\mathrm{N}$, Nicolaides-Bouman A. UK smoking statistics (2nd edn). Oxford: Oxford University Press, 1991

13 Emmanuel SC, Chan AS, Phe A. Cigarette smoking in Singapore. Singapore Med f 1988; 29: 119-24.

14 National Health Survey, 1992. Ministry of Health, Singapore, 1993.

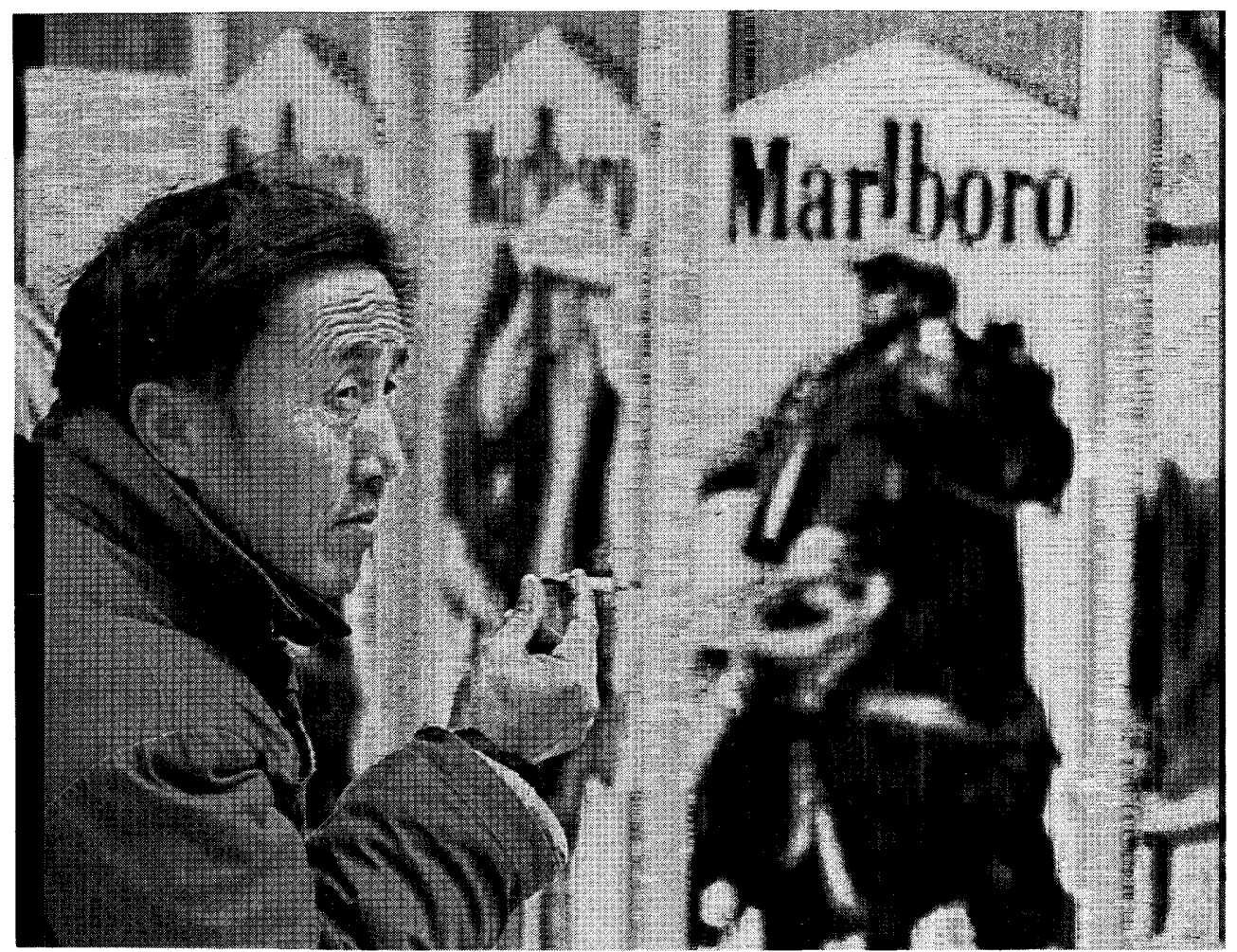

Philip Morris moving into Asia 